\title{
ON 4-DIMENSIONAL ELATION LAGUERRE PLANES ADMITTING SIMPLE LIE GROUPS OF AUTOMORPHISMS
}

\author{
GÜNTER F. STEINKE \\ Department of Mathematics \& Statistics \\ University of Canterbury, Private Bag 4800 \\ Christchurch, New Zealand
}

No. 159

December, 1997

To appear in Forum Mathematicum 


\title{
ON 4-DIMENSIONAL ELATION LAGUERRE PLANES ADMITTING SIMPLE LIE GROUPS OF AUTOMORPHISMS
}

\author{
GÜNTER F. STEINKE \\ Department of Mathematics \\ University of Canterbury \\ Private Bag 4800 \\ Christchurch, New Zealand
}

\begin{abstract}
This paper concerns 4-dimensional (topological locally compact connected) elation Laguerre planes that admit large automorphism groups. In particular, it is shown that such a plane is classical if its automorphism group is at least 11dimensional. Furthermore, the elation Laguerre planes admitting simple Lie groups of automorphisms are investigated and various characterizations of the classical complex Laguerre plane and the semi-classical Laguerre planes are obtained.
\end{abstract}

\section{Introduction}

A Laguerre plane $\mathcal{L}=(P, \mathcal{C}, \|)$ is an incidence structure consisting of a point set $P$, a circle set $\mathcal{C}$ and an equivalence relation $\|$ (parallelism) defined on the point set such that three mutually non-parallel points can be joined by a unique circle, such that the circles which touch a fixed circle $K$ at $p \in K$ partition $P \backslash|p|$, such that each parallel class meets each circle in a unique point (parallel projection), and such that there is a circle that contains at least three points (compare [10] and [11]).

When the circle sets are topologized by the Hausdorff metric with respect to a metric that induces the topology of the point set, then the planes are topological in the sense that the operations of joining three points by a circle, intersecting of two circles, and touching are continuous with respect to the induced topologies on their respective domains of definition. A topological Laguerre plane is called locally compact, connected, or finite-dimensional if the point space has the respective topological property. For brevity, a locally compact connected finite-dimensional topological Laguerre plane will be called a finite-dimensional Laguerre plane. According to $[15,2.3]$ a finite-dimensional Laguerre plane can only be of dimension 2 or 4 .

1991 Mathematics Subject Classification. 51H15.

Typeset by $\mathcal{A} \mathcal{M} S-\mathrm{T}_{\mathrm{E}} \mathrm{X}$ 
The classical 2- or 4-dimensional Laguerre plane is obtained as the geometry of non-trivial plane sections of an elliptic cone in real or complex projective 3-dimensional space, respectively, with its vertex removed. The classical real Laguerre plane can be generalized to 2-dimensional ovoidal Laguerre planes where one similarly takes a cone over an oval in the real projective plane and considers its nontrivial plane sections.

There are many models of 2-dimensional Laguerre planes known, for example see [17] and [1] for a large class of planes. In [29] we constructed a family of 4-dimensional Laguerre planes, see 2.11 for an explicit description of these planes. These semi-classical Laguerre planes were the first non-classical 4-dimensional Laguerre planes. They are obtained by pasting together two halves of the classical complex Laguerre plane along a 3-dimensional separating set in the point set. In the present paper we give a characterization of the semi-classical Laguerre planes in terms of their automorphism groups and how they act on the set of parallel classes and on the circle set.

\section{The topology and geometry of 4-dimensional Laguerre planes}

2.1. Associated with every point $p$ of $\mathcal{L}$ there is a derived incidence structure, called the derived affine plane $\mathcal{A}_{p}=\left(A_{p}, \mathcal{L}_{p}\right)$ at $p$, whose point set $A_{p}$ consists of all points of $\mathcal{L}$ that are not parallel to $p$ and whose line set $\mathcal{L}_{p}$ consists of all restrictions to $A_{p}$ of circles of $\mathcal{L}$ passing through $p$ and of all parallel classes not passing through $p$. Indeed, $\mathcal{L}$ is a Laguerre plane if and only if all incidence structures $\mathcal{A}_{p}$ are affine planes. It can easily be seen that derived affine planes of the classical Laguerre plane are even topological locally compact connected affine planes. This, in fact, is true for every finite-dimensional Laguerre plane. Furthermore, the projective extension $\mathcal{P}_{p}$ of $\mathcal{A}_{p}$ can be made to a topological locally compact connected projective plane. We call $\mathcal{P}_{p}$ the derived projective plane at $p$.

Circles not passing through the point $p$ of derivation induce closed ovals in $\mathcal{P}_{p}$ by removing the point parallel to $p$ and adding in $\mathcal{P}_{p}$ the infinite point of the lines that come from parallel classes of $\mathcal{L}$. The line at infinity of $\mathcal{P}_{p}$ (relative to $\mathcal{A}_{p}$ ) is a tangent to this oval.

2.2. According to $[15,2.5]$ the classical complex Laguerre plane can be characterized in terms of a single derivation.

Theorem. A 4-dimensional Laguerre plane is isomorphic to the classical complex Laguerre plane if and only if at least one derived affine plane is Desarguesian.

This is due to a remarkable result of T. Buchanan [5] which says that the only topological ovals in the Desarguesian projective complex plane are conics.

2.3. Circles and parallel classes of 4-dimensional Laguerre planes are homeomorphic to the 2-sphere $\mathbb{S}_{2}$ and to $\mathbb{R}^{2}$, respectively. Furthermore, the space $\Pi$ of all parallel classes can be identified with a circle and thus is homeomorphic to $\mathbb{S}_{2}$. The circle set $\mathcal{C}$ is homeomorphic to the direct product of three parallel classes, that is, $\mathcal{C}$ is 
homeomorphic to $\mathbb{R}^{6}$. The common point set of 4-dimensional Laguerre planes is a metrizable 4-dimensional topological space. More precisely, given a circle in a 4-dimensional Laguerre plane $\mathcal{L}$ one can consider the point space as a fibre bundle over this circle with fibres being the parallel classes. The projection map of the bundle is given by the parallel projection in the Laguerre plane. Since the point space of a 4-dimensional affine plane is the direct product of two lines, it follows that the point space of $\mathcal{L}$ is a locally trivial fibre bundle over $\mathbb{S}_{2}$ with fibres homeomorphic to $\mathbb{R}^{2}$. Considering the characteristic map of the bundle, which can be related to the function describing some circle, it was shown in [31] that the point space of a 4dimensional Laguerre plane is equivalent to the point space of the classical complex Laguerre plane in the category of fibre bundles over $\mathbb{S}_{2}$ with fibres homeomorphic to $\mathbb{R}^{2}$. Furthermore, the point space of the classical complex Laguerre plane is bundle-equivalent to the tangent bundle of the 2-sphere $\mathbb{S}_{2}$.

For the intersection of circles the following result was obtained in [11, Satz 3.3.b] and $[6$, Satz 3.7.b].

Theorem. In a 4-dimensional Laguerre plane any two circles have at least one point in common.

2.4. The collection of all continuous automorphisms of a 4-dimensional Laguerre plane $\mathcal{L}$ forms a group with respect to composition, the automorphism group $\Gamma$ of $\mathcal{L}$. This group is a Lie group with respect to the compact-open topology, see $[8$, Satz 3.9] or [27]. The kernel of a Laguerre plane consists of all automorphisms that fix each parallel class. This collection of automorphisms is a closed normal subgroup of the automorphism group of the Laguerre plane. Furthermore, the connected component of the identity in the kernel is solvable, cf. [30, Corollary 3.6].

2.5. The topological dimensions of the stabilizer $G_{x}$ and the orbit $G(x)$ of a Lie group $G$ acting on a manifold $X$ are related by the dimension formula $\operatorname{dim} G=$ $\operatorname{dim} G_{x}+\operatorname{dim} G(x)$ for $x \in X$, cf. [12]. As for the stabilizer of certain points in a Laguerre plane one has the following lemma.

Lemma. Let $F$ be a set of four non-concircular points with at least three of them being mutually non-parallel. The stabilizer of $F$ in the automorphism group contains at most two automorphisms. The stabilizer of $F$ in the kernel $T$ is trivial.

This lemma and the dimension formula readily yield upper bounds for the dimension of the automorphism group and kernel of a 4-dimensional Laguerre plane.

Theorem. The dimension of the automorphism group of a 4-dimensional Laguerre plane is at most 14 and the kernel has dimension at most 8.

These dimensions are attained in the classical complex Laguerre plane: The kernel is isomorphic to $\mathbb{C} \backslash\{0\} \ltimes \mathbb{C}^{3}$ and the connected component of the identity in the automorphism group of the classical 4-dimensional Laguerre plane is isomorphic to $\left(\mathbb{C} \backslash\{0\} \times \mathrm{SO}_{3}(\mathbb{C})\right) \ltimes \mathbb{C}^{3}$.

2.6. The automorphism group of a 4-dimensional Laguerre plane operates on the set $\Pi$ of all parallel classes in a canonical way and likewise the stabilizer of a circle 
$K$ operates on $K$. Since both $\Pi$ and $K$ are homeomorphic to $\mathbb{S}_{2}$, we state a few results about transformation groups of $\mathbb{S}_{2}$, which we shall use time and again in the following sections.

Theorem. Let $G$ be a locally compact, connected transformation group of $\mathbb{S}_{2}$.

(1) Every closed $G$-orbit in $\mathbb{S}_{2}$ is a manifold and $G$ always has a closed $G$-orbit, that is, $G$ fixes a point, has an orbit that is homeomorphic to the 1-sphere $\mathbb{S}_{1}$ or acts transitively on $\mathbb{S}_{2} ; c f$. [13, Satz 1,2$]$.

(2) If $G$ acts transitively and effectively on $\mathbb{S}_{2}$, then $G$ is isomorphic and acts equivalently to $P S L_{3}(\mathbb{R}), P S L_{2}(\mathbb{C})$ or $S O_{3}(\mathbb{R})$ in their respective standard actions on $\mathbb{S}_{2} ; c f .[19, \S 10]$.

(3) If $G$ acts doubly transitively and effectively on $\mathbb{S}_{2}$, then $G$ is isomorphic and acts equivalently to $P S L_{2}(\mathbb{C})$ in its standard action on $\mathbb{S}_{2} ;$ cf. $[35 ; 36]$.

For transitive and effective actions one $\mathbb{S}_{1}$ on has the following.

(4) A locally compact, connected, transitive and effective transformation group of $\mathbb{S}_{1}$ is isomorphic and acts equivalently to the rotation group $\mathrm{SO}_{2}(\mathbb{R})$ or to a finite covering group of $P S L_{2}(\mathbb{R}) ; c f .[4]$ or $[21,3.18]$.

2.7. An elation Laguerre plane is a Laguerre plane that admits a subgroup in the kernel, called the elation group, which acts sharply transitively on the set of circles. The name elation group comes from the fact that an automorphism in this group induces an elation in the derived projective plane at each of its fixed points. Furthermore, these automorphisms induce elations in the associated Lie geometry and one obtains an elation generalized quadrangle; cf. [26] for generalized quadrangles and their relation to Laguerre planes and the others types of circle planes.

For 4-dimensional Laguerre planes, the collection $\Delta$ of all automorphisms in the kernel $T$ that fix no circle plus the identity is a (closed) normal subgroup of $T$. Furthermore, in the case of elation Laguerre planes, $\Delta$ acts sharply transitively on the set of circles, see [30]. So $\Delta$ is the elation group in this case. The 2-dimensional elation Laguerre planes are precisely the ovoidal planes by [32, Lemma 2.8]. With this definition of $\Delta$ we obtained in [30] the following characterization of 4-dimensional elation Laguerre planes; cf. [16].

Theorem. For a 4-dimensional Laguerre plane $\mathcal{L}$ let $\Delta$ be the collection of all automorphisms in the kernel $T$ that fix no circle plus the identity. Then $\Delta$ is a closed normal subgroup of the automorphism group of $\mathcal{L}$ and the following are equivalent:

(1) $\mathcal{L}$ is an elation Laguerre plane;

(2) the kernel $T$ is circle transitive;

(3) $T$ is at least 7 -dimensional;

(4) $\Delta$ is 6-dimensional;

(5) $\Delta$ is connected and isomorphic to $\mathbb{R}^{6}$;

(6) $\Delta$ is sharply transitive on the set of circles. 
2.8. Each derived projective plane of an elation Laguerre plane is a dual translation plane with centre $\omega$ (the infinite point of lines that come from parallel classes of the Laguerre plane). The stabilizer of a circle is linearly represented on $\Delta$ by conjugation. Therefore, the well developed theory of translation planes and, if the stabilizer of a circle is large enough, the representation theory of Lie groups can be applied to classify the most homogeneous elation Laguerre planes. This was carried out in [28] and all 4-dimensional elation Laguerre planes with a 4-dimensional stabilizer of a circle were classified. We shall see that the semiclassical 4-dimensional Laguerre planes are precisely those planes with no fixed parallel class and reducible action of a 3-dimensional complement of the kernel in the stablizer of a circle on $\Delta$.

2.9. Löwen [16] gave a reinterpretation of elation Laguerre planes as pseudo-ovoidal Laguerre planes which naturally generalizes the construction of ovoidal Laguerre planes. His construction uses the Grassmannian $G_{n, m}(\mathbb{R})$, that is, the collection of all $m$-dimensional subspaces of the $n$-dimensional real vector space $\mathbb{R}^{n}$ where $m$ and $n$ are positive integers such that $m<n$. The Grassmannian $G_{n, m}(\mathbb{R})$ can be made into a compact connected manifold. A set $\mathcal{O} \subseteq G_{3 m, m}(\mathbb{R})$ of $m$-dimensional subspaces of $\mathbb{R}^{3 m}$ is called a pseudo-oval if for every element $p \in \mathcal{O}$ there is a $2 m$ dimensional subspace $t_{p}$ ('tangent') such that $t_{p}$ and the collection of all joins in $G_{3 m, m}(\mathbb{R})$ of $p$ with elements of $\mathcal{O}$ distinct from $p$ induce a planar spread in the factor space $\mathbb{R}^{3 m} / p$. A topological pseudo-oval is one where the map

$$
\mathcal{O} * \mathcal{O} \rightarrow G_{3 m, 2 m}(\mathbb{R}):\{p, q\} \mapsto \begin{cases}p \vee q, & \text { if } p \neq q \\ t_{p}, & \text { if } p=q\end{cases}
$$

is a homeomorphism onto its image.

A pseudo-oval can be regarded as a sufficiently large family of translation planes sitting on different $2 m$-dimensional subspaces of one $\mathbb{R}^{3 m}$ such that the intersection of any two translation planes is a line in both of them. Finite analogues were studied in [34], [20] and [7]. For $m=1$ one exactly obtains a topological oval in the real projective plane. The other interesting case is $m=2$ which yields 4 -dimensional elation Laguerre planes.

2.10. Matrix representation of 2-dimensional pseudo-ovals and 4-dimensional elation Laguerre planes. For each $z \in \mathbb{S}_{2} \simeq \mathbb{R}^{2} \cup\{\infty\}$ let

$$
D(z)=\left(\begin{array}{l}
A(z) \\
B(z) \\
C(z)
\end{array}\right)
$$

be a $6 \times 2$ matrix with $2 \times 2$ matrices $A, B, C$ such that $D(\infty)=\left(\begin{array}{lll}I & 0 & 0\end{array}\right)^{t}$, where ${ }^{t}$ denotes the transpose of the matrix and where 0 and $I$ denote the $2 \times 2$ zero and identity matrix, respectively, and such that for all $z \in \mathbb{R}^{2}$ the second row of $B(z)$ equals $z$ and $C(z)=I$. One can further assume that $A(0)=B(0)=0$.

Each compact 2-dimensional pseudo-oval can be represented by such a mapping $D$. Moreover, $D$ is continuous in $\mathbb{R}^{2}$ and $D(z) A(z)^{-1}$ tends to $D(\infty)$ for $z \rightarrow$ 
$\infty$. The 2-dimensional subspaces generated by the rows of $D(z)$ correspond to the elements of the pseudo-oval.

Circles of the associated elation Laguerre plane $\mathcal{L}$ are of the form

$$
K_{c}=\left\{(z, c \cdot D(z)) \mid z \in \mathbb{S}_{2}\right\}
$$

for every $c \in \mathbb{R}^{6}$. The elation group $\Delta$ of $\mathcal{L}$ is given by all maps

$$
(z, w) \mapsto(z, w+c \cdot D(z))
$$

for $c \in \mathbb{R}^{6}$; the connected component of the identity in the kernel contains all maps

$$
(z, w) \mapsto(z, r w+c \cdot D(z))
$$

for $c \in \mathbb{R}^{6}, r \in \mathbb{R}, r>0$. The continuity of the geometric operations in such a Laguerre plane described by a matrix valued mapping $D$ reduces to the continuity of $D$ in $\mathbb{R}^{2}$ and to $\lim _{z \rightarrow \infty} D(z) A(z)^{-1}=D(\infty)$, cf. [30, Proposition 5.8].

2.11 Example. The semi-classical Laguerre planes from [29] can be described as follows. For $q \in \mathbb{R}, 0 \leq q<1$ let $f_{q}: \mathbb{C} \rightarrow \mathbb{C}$ be the mapping defined by $f_{q}(z)=z+q \bar{z}$ where $\bar{z}$ denotes the complex conjugate of $z$. This mapping is an $\mathbb{R}$-linear permutation of $\mathbb{C}$ whose inverse is given by $f_{q}^{-1}(z)=\frac{1}{1-q^{2}}(z-q \bar{z})$. The point set of the semi-classical Laguerre plane $\mathcal{L}_{q}$ is $P=(\mathbb{C} \cup\{\infty\}) \times \mathbb{C}$. Two points $(z, w)$ and $\left(z^{\prime}, w^{\prime}\right)$ are parallel if and only if $z=z^{\prime}$. Circles of $\mathcal{L}_{q}$ are of the form

$$
\begin{aligned}
K_{a_{2}, a_{1}, a_{0}} & =\left\{(z, w) \in \mathbb{C} \times \mathbb{C} \mid w=a_{2} z^{2}+a_{1} z+a_{0}, \operatorname{Im}(z) \geq 0\right\} \\
& \cup\left\{(z, w) \in \mathbb{C} \times \mathbb{C} \mid w=f_{q}^{-1}\left(f_{q}\left(a_{2}\right) z^{2}+f_{q}\left(a_{1}\right) z+f_{q}\left(a_{0}\right)\right), \operatorname{Im}(z) \leq 0\right\} \\
& \cup\left\{\left(\infty, a_{2}\right)\right\}
\end{aligned}
$$

for $a_{0}, a_{1}, a_{2} \in \mathbb{C}$. Clearly, we obtain the classical complex Laguerre plane for $q=0$.

On each halfplane $P_{+}=\{(z, w) \in P \mid \operatorname{Im}(z) \geq 0$ or $z=\infty\}$ and $P_{-}=\{(z, w) \in$ $P \mid \operatorname{Im}(z) \leq 0$ or $z=\infty\}$ the geometry and topology of $\mathcal{L}_{0}$ is induced. The two halves are glued together along $P_{+} \cap P_{-}=\{(z, w) \in P \mid z \in \mathbb{R} \cup\{\infty\}\}$ using the mapping $f_{q}$. Two Laguerre planes $\mathcal{L}_{q}$ and $\mathcal{L}_{q^{\prime}}$ are isomorphic if and only if $q=q^{\prime}$.

These Laguerre planes can be represented in the form of 2.10 as follows. We identify $z=x+i y \in \mathbb{C}$ for $x, y \in \mathbb{R}$ with the pair $(x, y)$. For $0<p \leq 1$ let

$$
\begin{array}{ll}
A(x, y)=\left(\begin{array}{cc}
y^{2}-x^{2} & -2 x y \\
2 x y & y^{2}-x^{2}
\end{array}\right), \quad B(x, y)=\left(\begin{array}{cc}
y & -x \\
x & y
\end{array}\right), \quad \text { for } y \geq 0 \\
A(x, y)=\left(\begin{array}{cc}
p y^{2}-x^{2} & -2 x y \\
2 p x y & p y^{2}-x^{2}
\end{array}\right), \quad B(x, y)=\left(\begin{array}{cc}
p y & -x \\
x & y
\end{array}\right), \quad \text { for } y \leq 0 .
\end{array}
$$

Every semi-classical Laguerre plane is isomorphic to an elation Laguerre plane as described above for exactly one $p, 0<p \leq 1$. (In fact, $p$ and $q$ are related by $p=\left(\frac{1-q}{1+q}\right)^{2}$.) One obtains the classical complex Laguerre plane for $p=1$. 
Besides the automorphisms every elation Laguerre plane admits, the semi-classical Laguerre planes further admit the following automorphisms

$$
(z, w) \mapsto \begin{cases}\left(\frac{a z+b}{c z+d}, \frac{w}{(c z+d)^{2}}\right), & \text { for } \operatorname{Im}(z) \geq 0, c z+d \neq 0 \\ \left(\frac{a z+b}{c z+d}, f_{q}^{-1}\left(\frac{f_{q}(w)}{(c z+d)^{2}}\right)\right), & \text { for } \operatorname{Im}(z) \leq 0, c z+d \neq 0 \\ \left(\infty, c^{2} w\right), & \text { for } z \in \mathbb{C}, c z+d=0 \\ \left(\frac{a}{c}, \frac{w}{c^{2}}\right), & \text { for } z=\infty, c \neq 0 \\ \left(\infty, d^{2} w\right), & \text { for } z=\infty, c=0\end{cases}
$$

for all $a, b, c, d \in \mathbb{R}, a d-b c=1$. The collection of all these automorphisms forms a group isomorphic to the simple Lie group $\mathrm{PSL}_{2}(\mathbb{R})$. Together with the 7-dimensional group in the kernel from 2.10 such a semi-classical Laguerre plane thus has an automorphism group of dimension at least 10. In fact, the automorphism group of $\mathcal{L}_{q}$ is 10-dimensional unless $q=0$. In the latter case, we have the classical complex Laguerre plane, whose automorphism group is 14-dimensional.

\section{Elation Laguerre planes admitting automorphism groups of dimension at least 11}

We begin with a characterization of the classical complex Laguerre plane via its kernel, see $[8,5.2]$ or [30, Proposition 2.8], and the classification of 4-dimensional projective planes admitting large automorphism groups.

3.1. Theorem. A 4-dimensional Laguerre plane is isomorphic to the classical complex Laguerre plane if and only if the kernel $T$ is 8-dimensional.

Note that this theorem and Theorem 2.7 imply that a proper elation Laguerre plane has a 7-dimensional kernel (and 6-dimensional elation group).

3.2. Theorem. Let $\mathcal{P}$ be a 4-dimensional compact projective plane.

(1) If $\mathcal{P}$ admits a collineation group of dimension at least 9 , then $\mathcal{P}$ is Desarguesian; cf. [22; 23; 2, Satz 4] or [25, Theorem 72.8].

(2) If $\mathcal{P}$ admits an 8-dimensional collineation group that fixes a flag $(p, L)$, then $\mathcal{P}$ is Desarguesian or $\mathcal{P}$ is isomorphic to Betten's translation plane $\mathcal{B}$, see [3, Satz 2.b] for this plane, or to the dual $\mathcal{B}^{\prime}$ of $\mathcal{B} ;$ cf. $[24, \S 1,2]$ or $[25$, \$73]. In the latter two cases, the full collineation group is 8-dimensional and solvable and acts transitively on the line pencil through $p$ minus the line L. Moreover, the group of all central collineations with centre $p$ is 3-or 5-dimensional for $\mathcal{B}$ or $\mathcal{B}^{\prime}$, respectively.

Proof. We just verify the last two statements of (2). We coordinatise $\mathcal{B}$ in such a way that $p$ is the infinite point $\omega$ and $L$ is the infinite line and that the lines through $\omega$ other than $L$ become the verticals $L_{c}=\left\{(c, w) \mid w \in \mathbb{R}^{2}\right\}$ for $c \in \mathbb{R}^{2}$. According 
to $[3$, Satz 2.b] or $[25,73.10]$ the non-vertical lines of $\mathcal{B}$ are represented on the affine plane obtained by deleting $L$ as

$$
\left\{\left(z, z\left(\begin{array}{cc}
t-s^{2} & -\frac{1}{3} s^{3} \\
s & t
\end{array}\right)+c\right) \mid z \in \mathbb{R}^{2}\right\}
$$

for $s, t \in \mathbb{R}, c \in \mathbb{R}^{2}$. The connected component of the identity in the collineation group of $\mathcal{B}$ consists of all transformations of the form

$$
\mathbb{R}^{4} \rightarrow \mathbb{R}^{4}: v \mapsto v\left(\begin{array}{cccc}
r & r s & r\left(\frac{1}{2} s^{2}+t\right) & r s\left(\frac{1}{6} s^{2}+t\right) \\
0 & d r & d r s & d r\left(\frac{1}{2} s^{2}+t\right) \\
0 & 0 & d^{2} r & d^{2} r s \\
0 & 0 & 0 & d^{3} r
\end{array}\right)+v_{0}
$$

for $d, r, s, t \in \mathbb{R}, d, r>0, v_{0} \in \mathbb{R}^{4}$. In particular, a vertical line $L_{c}$ is taken under such a collineation to the line $L_{c^{\prime}}$ where $c^{\prime}=c\left(\begin{array}{cc}r & r s \\ 0 & d r\end{array}\right)+c_{0}$. This shows that the collineation group of $\mathcal{B}$ acts transitively on the set of affine vertical lines. The collineation from above is a central collineation with centre $\omega$ if and only if $r=d=1, s=0$ and the first two components of $v_{0}$ are zero. This leaves a 3-dimensional group of central collineations; cf. [25, Lemma 73.7.a].

As for $\mathcal{B}^{\prime}$ we can dualise the above description of $\mathcal{B}$ in such a way that the infinite line of $\mathcal{B}$ becomes the infinite point $\omega^{\prime}$ of $\mathcal{B}^{\prime}$ and the point $\omega$ becomes the infinite line of $\mathcal{B}^{\prime}$. Then the line pencil in $\mathcal{B}^{\prime}$ through $p$ minus the line $L$ corresponds in $\mathcal{B}$ to the points of $L \backslash\{p\}$ and the central collineations of $\mathcal{B}^{\prime}$ with centre $p$ are precisely the axial collineations of $\mathcal{B}$ with axis the infinite line $L$. By direct computation one now finds that the collineation group of $\mathcal{B}^{\prime}$ acts transitively on the line pencil through $p$ minus the line $L$ (e.g., the group obtained for $r=d=1, v_{0}=0$ as above); see also [25, Lemma 73.6]. That the group of central collineations with centre $p$ is 5-dimensional follows from the fact that the collineations that every translation plane admits, that is, the translations $v \mapsto v+v_{0}$ for $v_{0} \in \mathbb{R}^{4}$ and the homotheties $v \mapsto r v$ for $r \in \mathbb{R}, r \neq 0$, are axial collineations of $\mathcal{B}$ with axis the infinite line.

3.3. Lemma. Let $\mathcal{L}$ be a 4-dimensional Laguerre plane and let $\Sigma$ be a closed subgroup of the automorphism group of $\mathcal{L}$. Let $p$ be a point of $\mathcal{L}$ such that the stabilizer $\Sigma_{p}$ is at least 8-dimensional. Then $\mathcal{L}$ is classical or $\Sigma_{p}$ acts transitively on $\Pi \backslash|p|$.

Proof. By Theorem 3.2.2 the derived plane at $p$ is either Desarguesian or isomorphic to Betten's translation plane $\mathcal{B}$ or its dual $\mathcal{B}^{\prime}$. In the first case, $\mathcal{L}$ is classical by Theorem 2.2. In the other two cases, $\Sigma_{p}$ induces a group of collineations in the derived projective plane at $p$. Since the verticals in $\mathcal{B}$ as well as in $\mathcal{B}^{\prime}$ correspond to the parallel classes of $\mathcal{L}$ other that $|p|$, Theorem 3.2 .2 yields the transitivity of $\Sigma_{p}$ on $\Pi \backslash|p|$ in these cases. 
3.4. Corollary. Let $\mathcal{L}$ be a 4-dimensional Laguerre plane and let $\Sigma$ be a closed connected subgroup of the automorphism group of $\mathcal{L}$. If $\Sigma$ is at least 11-dimensional, then $\mathcal{L}$ is classical or $\Sigma$ acts transitively on the space $\Pi$ of all parallel classes.

Proof. We assume that $\Sigma$ is not transitive on $\Pi$. Then $\Sigma$ fixes a parallel class or has a 1-dimensional orbit in $\Pi$ by $[13$, Satz 1,2]. In the former case, one has $\operatorname{dim} \Sigma_{p} \geq 9$ for each point $p$ in the fixed parallel class. Hence the derived plane at $p$ is Desarguesian by Theorem 3.2 .1 and $\mathcal{L}$ is classical by Theorem 2.2. In the latter case, $\operatorname{dim} \Sigma_{p} \geq 8$ for each point on a parallel class in the 1-dimensional orbit. However, $\Sigma_{p}$ is not transitive on $\Pi \backslash|p|$ and so $\mathcal{L}$ must be classical by Lemma 3.3.

3.5. Theorem. Let $\mathcal{L}$ be a 4-dimensional Laguerre plane that admits an automorphism group of dimension at least 12. Then $\mathcal{L}$ is classical.

Proof. Let $\Sigma$ be a closed connected subgroup of the automorphism group $\Gamma$ of $\mathcal{L}$ and assume that $\operatorname{dim} \Sigma \geq 12$. Then $\operatorname{dim} \Sigma_{p} \geq 8$ for each point of $\mathcal{L}$. By Theorem 3.2 .2 the derived plane at $p$ is either Desarguesian or isomorphic to Betten's translation plane $\mathcal{B}$ or its dual $\mathcal{B}^{\prime}$. In the first case $\mathcal{L}$ is classical by Theorem 2.2 .

We show that neither $\mathcal{B}$ nor $\mathcal{B}^{\prime}$ can occur. We assume to the contrary that every derived plane is isomorphic to either $\mathcal{B}$ or $\mathcal{B}^{\prime}$. In particular, $\Sigma$ must be 12 dimensional and must act transitively on $\Pi$ by Corollary 3.4. Furthermore, $\Sigma_{p}$ is transitive on $\Pi \backslash|p|$. This shows that $\Sigma$ is 2-transitive on $\Pi$. The quotient group $\Sigma /(\Sigma \cap T)$, where $T$ is the kernel of $\mathcal{L}$, acts effectively on $\Pi$ and so $\Sigma /(\Sigma \cap T)$ is isomorphic and acts equivalently to $\mathrm{PSL}_{2}(\mathbb{C})$ in its standard action on $\mathbb{S}_{2}$ by Theorem 2.6.3. Hence $\Sigma \cap T$ is 6-dimensional.

The transitivity of $\Delta$ on the set of circles and of $\Sigma$ on $\Pi$ implies that $\Sigma$ acts transitively on the points of $\mathcal{L}$. Therefore $\Sigma \cap T$ has the same system of orbits in each parallel class. It follows from Lemma 2.5 that $\Sigma \cap T$ is transitive on each parallel class and thus $\operatorname{dim} \Sigma_{p} \cap T=4$ for each point $p$. In the derived projective plane at $p$, the group $\Sigma_{p} \cap T$ induces precisely those central collineations with centre the infinite point of the lines that come from parallel classes. However, the corresponding group is 3-dimensional in $\mathcal{B}$ and 5-dimensional in $\mathcal{B}^{\prime}$ by Theorem 3.2.2. This contradicition shows that neither $\mathcal{B}$ nor $\mathcal{B}^{\prime}$ can occur as derived planes.

3.6. Theorem. Let $\mathcal{L}$ be a 4-dimensional elation Laguerre plane that admits an automorphism group of dimension at least 11. Then $\mathcal{L}$ is classical.

Proof. By Theorem 3.5 we only have to consider the case that the automorphism group $\Gamma$ of $\mathcal{L}$ is 11-dimensional. In particular, we assume that $\mathcal{L}$ is not classical. Since $\mathcal{L}$ is an elation Laguerre plane, we know by Theorem 2.7 that the kernel $T$ is at least 7 -dimensional. Hence $\operatorname{dim} \Gamma / T \leq 4$. But $\Gamma / T$ acts transitively and effectively on $\Pi \simeq \mathbb{S}_{2}$ by Corollary 3.4 and so the connected component of the identity in this quotient group must be isomorphic to $\mathrm{PSL}_{3}(\mathbb{R}), \mathrm{PSL}_{2}(\mathbb{C})$ or $\mathrm{SO}_{3}(\mathbb{R})$ by Theorem 2.6.2. In particular, $\Gamma / T$ must be of dimension 3,6 or 8 , respectively. In our situation only dimension 3 can occur. Hence $\operatorname{dim} T=8$ and $\mathcal{L}$ is classical by Theorem 3.1 in contradiction to $\Gamma$ being 11-dimensional. 
3.7. Remark. The above Theorem remains valid for 4-dimensional Laguerre planes (not necessarily elation Laguerre planes); cf. [8, 7.4] and [28, §4]. One considers an 11-dimensional closed connected subgroup $\Sigma$ of the automorphism group of a 4-dimensional Laguerre plane $\mathcal{L}$. By Corollary 3.4, $\Sigma$ acts transitively on $\Pi$ or $\mathcal{L}$ is classical. In the former case $\Sigma /(T \cap \Sigma)$ is isomorphic to $\mathrm{PSL}_{3}(\mathbb{R}), \mathrm{PSL}_{2}(\mathbb{C})$ or $\mathrm{SO}_{3}(\mathbb{R})$ by Theorem 2.6.2. One then excludes the first two cases. In the last remaining case the kernel $T$ is 8 -dimensional and $\mathcal{L}$ is classical by Theorem 3.1.

\section{Subgroups in the elation complement of elation Laguerre planes}

In this section $\mathcal{L}=(P, \mathcal{C}, \|)$ always denotes a 4-dimensional elation Laguerre plane and $\Gamma, T$ and $\Delta \cong \mathbb{R}^{6}$ denote its automorphism group, kernel and elation group, respectively. For a subgroup $\Phi$ of $\Gamma$ we denote the connected component of the identity by $\Phi^{1}$. In this section we investigate the possible actions and isomorphism types of 3-dimensional groups whose intersection with $T$ is discrete.

4.1. We say that a subgroup $\Sigma$ of $\Gamma$ is in the elation complement if its intersection with $\Delta$ is trivial, that is, $\Sigma \cap \Delta=\{i d\}$. The following well-known group theoretic result shows that we can always assume that a group in the elation complement fixes a circle.

Lemma. Let $\Sigma$ be a subgroup of $\Gamma$ in the elation complement. Let $K$ be a circle. Then $\Sigma$ is isomorphic to a subgroup $\Sigma^{\prime}$ in the stabilizer of $K$. Furthermore, if the intersection of $\Sigma$ with $T$ is discrete, then so is the intersection of $\Sigma^{\prime}$ with $T$.

Proof. Since $\Delta$ is sharply transitive on $\mathcal{C}$, there is precisely one $\delta_{\sigma} \in \Delta$ for each $\sigma \in \Sigma$ such that $\delta_{\sigma} \sigma(K)=K$. Let

$$
\Sigma^{\prime}=\left\{\delta_{\sigma} \sigma \mid \sigma \in \Sigma\right\}
$$

Using the fact that $\Delta$ is normal, it is readily verified that $\Sigma^{\prime}$ is a subgroup of $\Gamma_{K}$. Since $\Sigma$ is in the elation complement, the map $\Sigma \rightarrow \Sigma^{\prime}: \sigma \mapsto \delta_{\sigma} \sigma$ is an isomorphism from $\Sigma$ onto $\Sigma^{\prime}$. Furthermore, $\Sigma^{\prime} \cap T$ is the image of $\Sigma \cap T$ under the above isomorphism.

A subgroup $\Sigma$ in the stabilizer of a circle $K$ operates on the circle set $\mathcal{C}$ and on the elation group $\Delta$ by conjugation. The operation on $\Delta$ yields a linear and faithful representation of $\Sigma$ on $\mathbb{R}^{6} \cdot \mathcal{C}$ and $\Delta$ can be identified in such a way that the disinguished circle $K$ corresponds to the identity. More precisely, $\delta \in \Delta$ is identified with $\delta(K)$. Then both actions are equivalent, that is, $\sigma \delta \sigma^{-1} \in \Delta$ is identified with $\sigma \delta(K)$ for $\sigma \in \Sigma$. In the representation 2.10 a circle $L$ has coordinate vector $c$ and this vector $c$ also describes the corresponding element of $\Delta$.

4.2. Lemma. Let $K$ be a circle and let $p, q, r$ be three distinct points on $K$. Let $\Sigma$ be a closed subgroup in the stabilizer of $K$ such that $\Sigma \cap T$ is discrete. Then the stabilizer $\Sigma_{p, q, r}$ in $\Sigma$ of these three points is 0-dimensional.

Proof. Let $p, q, r$ be three distinct points on the circle $K$. We assume that $\Sigma_{p, q, r}$ has dimension at least 1 . The derived projective plane $\mathcal{P}$ at $p$ is a dual translation 
plane by 2.8. Let $W$ be the infinite line of $\mathcal{P}$ and let $L$ be the line that comes from the circle $K$. In particular, $L$ does not pass through the translation centre $\omega$ of $\mathcal{P}$.

The group $\Sigma_{p, q, r}$ induces in $\mathcal{P}$ an at least 1-dimensional group of collineations that fixes the line $L$ and the points $q, r$ and the infinite point $\omega_{L}=L \cap W \neq \omega$ on it. Together with the 1-dimensional group induced by $T_{K}$ we have an at least 2-dimensional group of collineations of $\mathcal{P}$ that fixes the line $L$ and three of its points. According to the dualisation of [2, Lemma 6] the projective plane $\mathcal{P}$ must be Desarguesian. Hence $\mathcal{L}$ is classical by Theorem 2.2 .

For the classical complex Laguerre plane one has that $\Gamma^{1} / T \cong \operatorname{PSL}_{2}(\mathbb{C})$ and $\Gamma_{p, q, r}^{1} /\left(\Gamma_{p, q, r}^{1} \cap T\right)=\{i d\}$. Since $\Sigma_{p, q, r} /\left(\Sigma_{p, q, r} \cap T\right)$ is a subgroup of $\Gamma_{p, q, r}^{1} /\left(\Gamma_{p, q, r}^{1} \cap T\right)$ in a canonical way and because $\Sigma_{p, q, r} \cap T$ is discrete, we have a contradiction to our assumption $\operatorname{dim} \Sigma_{p, q, r} \geq 1$.

4.3. Lemma. Let $K$ be a circle and let $\Sigma$ be a closed, connected 3-dimensional subgroup of $\Gamma_{K}$ such that $\Sigma \cap T$ is discrete. Suppose that $\Sigma$ leaves a 1-or 2-dimensional vector subspace of $\mathcal{C}$ invariant. Then $\Sigma$ fixes a point on $K$.

Proof. Let $U$ be a 1 -dimensional $\Sigma$-invariant vector subspace of $\mathcal{C}$. This subspace is generated by a circle $L \neq K$. We represent $\mathcal{L}$ as in 2.10 such that $K$ and $L$ become the circles $K_{0}$ and $K_{c}$, respectively, for some $c \in \mathbb{R}^{6}, c \neq 0$. Then every circle in $U$ is of the form $K_{r c}$ for some $r \in \mathbb{R}$. From this fact we see that $L^{\prime} \cap K=L \cap K$ for every circle $L^{\prime} \in U, L^{\prime} \neq K$. Since $U$ is $\Sigma$-invariant, it follows that $L \cap K$ is invariant under $\Sigma$. However, $L \cap K$ is non-empty by Theorem 2.3 and contains at most two points. Therefore, $L \cap K$ consists of fixed points of $\Sigma$.

Now let $U$ be a 2-dimensional $\Sigma$-invariant vector subspace of $\mathcal{C}$. We assume to the contrary that $\Sigma$ fixes no point of $K$ and therefore $\operatorname{dim} \Sigma_{p} \leq 2$ for every point $p \in K$. The first part then yields that no 1 -dimensional vector subspace of $U$ is invariant under $\Sigma$. Hence every stabilizer of a 1-dimensional vector subspace $V$ of $U$ is 2-dimensional. As before it follows that $\Sigma_{V} \subseteq \Sigma_{L \cap K}$ for every circle $L \in V$, $L \neq K$. This shows that $\operatorname{dim} \Sigma_{p}=2$ for every point $p \in L \cap K$ and every circle $L \in U, L \neq K$ and that $\Sigma_{V}=\Sigma_{L \cap K}=\Sigma_{p}$ where $V$ is the 1-dimensional vector subspace of $\mathcal{C}$ generated by $L$.

We claim that $U$ cannot entirely consist of tangent circles to $K$. Obviously, $U$ cannot be in the tangent pencil of $K$ at a particular point $q \in K$ for otherwise this point $q$ would be fixed under $\Sigma$. There therefore are tangent circles $L_{1}$ and $L_{2}$ that touch $K$ in different points $p_{1}$ and $p_{2}$, respectively. We consider the derived projective plane $\mathcal{P}$ at $p_{1}$. In $\mathcal{P}$ the circles $K$ and $L_{1}$ of $\mathcal{L}$ induce lines $K^{\prime}$ and $L_{1}^{\prime}$, respectively. Both lines pass through the infinite point $\omega^{\prime}=K^{\prime} \cap W$ where $W$ is the line at infinity in $\mathcal{P}$. By 2.1 the circle $L_{2}$ induces a closed oval $L_{2}^{\prime}$ in $\mathcal{P}$ having $W$ as a tangent. By $[6,2.6]$ there are precisely two tangent lines to $L_{2}^{\prime}$ through $\omega^{\prime}$, namely $K^{\prime}$ and $W$. This shows that $L_{1}$ intersects $L_{2}$ in two points. We represent $\mathcal{L}$ as in 2.10 in such a way that $K$ has coordinate vector the zero vector. Let $c_{1}$ and $c_{2}$ be the non-zero coordinate vectors of the circles $L_{1}$ and $L_{2}$, respectively. Since $U$ is a vector subspace, the circle with coordinate vector $c_{1}-c_{2}$ belongs to $U$ and intersects $K$ in two points - a contradiction to our assumption.

Hence there is a circle $L \in U, L \neq K$ that intersects $K$ in precisely two points 
$p$ and $q$. From above we know that $\operatorname{dim} \Sigma_{p, q}=2=\operatorname{dim} \Sigma_{p}$. Hence $p$ has an 1-dimensional $\Sigma$-orbit in $K$. For every point $r \neq p, q$ in this orbit we thus have by the dimension formula that $\operatorname{dim} \Sigma_{p, q, r} \geq 1$ in contradiction to Lemma 4.2. This proves that $\Sigma$ must fix a point of $K$.

4.4. Proposition. The automorphism group of a 4-dimensional elation Laguerre plane $\mathcal{L}$ contains no subgroup isomorphic to Spin(3), the simply connected covering group of $\mathrm{SO}_{3}(\mathbb{R})$. In particular, every subgroup locally isomorphic to $\mathrm{SO}_{3}(\mathbb{R})$ is already isomorphic to $\mathrm{SO}_{3}(\mathbb{R})$.

Proof. Suppose that $\Phi$ is a subgroup of $\Gamma$ isomorphic to Spin(3). By Lemma 4.1 we may assume that $\Sigma$ fixes a circle $K$. Furthermore, $\Phi \cap T$ is discrete, since $\Phi$ is almost simple. $\Phi$ is linearly and faithfully represented on $\Delta \cong \mathbb{R}^{6}$. This representation is completely reducible by Weyl's theorem, i.e., $\Phi$ splits into the direct sum of irreducible $\Phi$-invariant subspaces; cf. [9, Theorem 35.4]. From [37] we find that the only irreducible and faithful representation of $\Phi$ in dimension at most 6 occurs in dimension 4 . (In fact, this is the representation of $\operatorname{Spin}(3) \cong \mathrm{SU}_{2}(\mathbb{C})$ on $\mathbb{C}^{2} \cong \mathbb{R}^{4}$.) This means that $\Phi$ must fix a 1-dimensional vector subspace of $\mathcal{C}$. Hence $\Phi$ has a fixed point on $K$ by Lemma 4.2. However, $\Phi /(\Phi \cap T)$ acts effectively on $\Pi \simeq \mathbb{S}_{2}$ and so $\Phi /(\Phi \cap T)$ is isomorphic to $\mathrm{SO}_{3}(\mathbb{R})$ and acts on $\Pi$ like the rotation group on $\mathbb{S}_{2}$ by [18, Theorem 6.7.1]. In particular, $\Phi$ acts transitively on $\Pi$ and thus on $K$ - a contradiction. This shows that Spin(3) cannot act as a group of automorphisms of $\mathcal{L}$.

4.5. Remark. The above Proposition remains valid for 4-dimensional Laguerre planes (not necessarily elation Laguerre planes); cf. [8, 4.4] and [28, Lemma 4.6].

4.6. Theorem. Let $\mathcal{L}$ be a 4-dimensional elation Laguerre plane. Let $K$ be a circle and let $\Sigma$ be closed connected 3-dimensional subgroup in the stabilizer of $K$ such that $\Sigma \cap T$ is discrete.

(1) If $\Sigma$ acts transitively on $K$, then $\Sigma$ is isomorphic to $\mathrm{SO}_{3}(\mathbb{R})$. In this case, $\Delta \cong \mathbb{R}^{6}$ splits into the direct sum of two 3-dimensional irreducible $\Sigma$-invariant subspaces.

(2) If $\Sigma$ is not transitive on $K$ but still acts fixed-point-free on $K$, then $\Sigma$ is isomorphic to either $P S L_{2}(\mathbb{R})$ or to $S L_{2}(\mathbb{R})$. In this case, $\Sigma$ has precisely three orbits on $K$, one 1-dimensional orbit $B_{1}$ homeomorphic to the 1-sphere $\mathbb{S}_{1}$ and the two connected components of $K \backslash B_{1}$. The effective operation of $\Sigma$ on $B_{1}$ is sharply 3-transitive and thus is equivalent to the standard operation of $P S L_{2}(\mathbb{R})$ on $\mathbb{S}_{1}$. In case $\Sigma \cong P S L_{2}(\mathbb{R})$, the elation group splits into the direct sum of two 3-dimensional irreducible $\Sigma$-invariant subspaces; in case $\Sigma \cong S L_{2}(\mathbb{R})$, the group $\Sigma$ acts irreducibly on $\Delta$ and the centre of $\Sigma$ acts trivially on $K$.

(3) If $\Sigma$ fixes a point $p$ on $K$, then either $\mathcal{L}$ is classical or the derived affine plane at each point $q \| p$ is isomorphic to Betten's dual translation plane $\mathcal{B}^{\prime}$. In the latter case, the automorphism group $\Gamma$ of $\mathcal{L}$ is 10-dimensional and solvable and acts transitively on the parallel class $|p|$ of $p$ and on $P \backslash|p| . \Sigma$ acts transitively on $K \backslash\{p\}$ and $p$ is a fixed point of $\Gamma_{K}$. 
Proof. (1) $\Sigma /(\Sigma \cap T)$ is 3-dimensional and acts effectively and transitively on $K \simeq$ $\mathbb{S}_{2}$. Hence $\Sigma /(\Sigma \cap T) \cong \mathrm{SO}_{3}(\mathbb{R})$ by Theorem 2.6.2. Since $\Sigma \cap T$ is discrete, $\Sigma$ is locally isomorphic to $\mathrm{SO}_{3}(\mathbb{R})$. Hence $\Sigma$ is isomorphic to $\mathrm{SO}_{3}(\mathbb{R})$ by Proposition 4.4.

$\Sigma$ is linearly and faithfully represented on $\Delta$. Such a representation is completely reducible by Weyl's theorem, i.e., $\Delta$ splits into the direct sum of irreducible $\Sigma$ invariant subspaces; cf. [9, Theorem 35.4]. According to [37] the irreducible and faithful representations of $\mathrm{SO}_{3}(\mathbb{R})$ in dimension at most 6 occur in dimensions 3 and 5 . Hence, by Lemma 4.3 and the transitivity of $\Sigma$ on $K, \Delta$ must split into the direct sum of two 3-dimensional irreducible $\Sigma$-invariant subspaces.

(2) By Theorem 2.6.1 the group $\Sigma$ has an orbit $B_{1}$ homeomorhic to $\mathbb{S}_{1}$ in $K$. The kernel $N$ of the operation of $\Sigma$ on $B_{1}$ is 0 -dimensional by Lemma 4.2. Hence $\Sigma / N$ is 3-dimensional and this factor group is isomorphic to a finite covering group of $\mathrm{PSL}_{2}(\mathbb{R})$ by Theorem 2.6.4. Therefore $\Sigma$ itself is locally isomorphic to $\mathrm{PSL}_{2}(\mathbb{R})$. However, $\Sigma$ is faithfully represented on $\Delta \cong \mathbb{R}^{6}$. Hence $\Sigma$ must be isomorphic to either $\mathrm{PSL}_{2}(\mathbb{R})$ or $\mathrm{SL}_{2}(\mathbb{R})$, cf. [37]. Furthermore, $\Sigma / N$ acts triply transitive on $B_{1}$ by Lemma 4.2 .

By the Jordan-Schönflies theorem $K \backslash B_{1}$ has precisely two connected components, each homeomorphic to $\mathbb{R}^{2}$. We assume that $\Sigma$ is not transitive on one of these components. Then, because $\Sigma$ is fixed-point-free by assumption, there is a point $p \in K \backslash B_{1}$ that has a 1 -dimensional orbit $B$. Since $\Sigma_{p}$ is 2-dimensional, this stabilizer of $p$ cannot be transitive on $B_{1}$ by Theorem 2.6.4. Hence there is a point $q \in B_{1}$ that is fixed under $\Sigma_{p}$ and so $\operatorname{dim} \Sigma_{p, q}=2$. Let $r \neq p$ be another point in $B_{1}$. Then $\operatorname{dim} \Sigma_{p, q, r} \geq 1$ in contradiction to Lemma 4.2. This shows that $\Sigma$ must be transitive on each connected component of $K \backslash B_{1}$.

As in (1) $\Delta$ is the direct sum of irreducible $\Sigma$-invariant subspaces. From [37] we find that the irreducible and faithful representations of $\mathrm{PSL}_{2}(\mathbb{R})$ and $\mathrm{SL}_{2}(\mathbb{R})$ in dimension at most 6 occur in dimensions 3 and 5 and in dimensions 2,4 , and 6 , respectively. As before, $\Delta$ must be the direct sum of two 3-dimensional irreducible $\Sigma$-invariant subspaces in case $\Sigma \cong \mathrm{PSL}_{2}(\mathbb{R})$. As for $\mathrm{SL}_{2}(\mathbb{R})$, only a 6-dimensional irreducible representation results in no invariant 1- or 2-dimensional subspace and thus no fixed points of $\Sigma$ on $K$. Furthermore, in this case, the centre of $\Sigma$ acts on $\mathcal{C}$ as a group of diagonal matrices. In particular, $L \cap K=\zeta(L) \cap K$ for $L \in \mathcal{C}$, $L \neq K$, and $\zeta$ in the centre of $\Sigma$. This shows that $\zeta$ acts trivially on $K$. Therefore $\Sigma / N \cong \mathrm{PSL}_{2}(\mathbb{R})$ and $\Sigma / N$ acts on $B_{1}$ like $\mathrm{PSL}_{2}(\mathbb{R})$ on $\mathbb{S}_{1}$ in its standard action.

(3) Since very point $q$ parallel to $p$ has an orbit of dimension at most 2 in the parallel class of $p$ and because the automorphism group $\Gamma$ of $\mathcal{L}$ is at least 10-dimensional, the dimension formula shows that the stabilizer $\Gamma_{q}$ is at least 8dimensional. According to Theorem 3.2 the derived projective plane $\mathcal{P}_{q}$ must be either Desarguesian or isomorphic to Betten's translation plane $\mathcal{B}$ or to its dual $\mathcal{B}^{\prime}$. In the first case $\mathcal{L}$ is classical by Theorem 2.2. The second case cannot occur since $\mathcal{P}_{q}$ is a dual translation plane by 2.8 and because a 4-dimensional translation plane that is also a dual translation plane must be Desarguesian; cf. [25, Corollary 64.15].

In the last case $\Gamma$ must be 10-dimensional and must be transitive on $|p|$. Thus every derived projective plane $\mathcal{P}_{q}$ at a point $q$ parallel to $p$ must be isomorphic to $\mathcal{B}^{\prime}$. Furthermore, $\Gamma_{p}$ operates transitively on $\Pi \backslash|p|$ by Lemma 3.3. Together with 
the elation group this implies that $\Gamma$ is transitive on $P \backslash|p|$. Since $\Sigma$ acts locally equivalent to $\Gamma_{p} /\left(\Gamma_{p} \cap T\right)$ on $\Pi$, we see that $\Sigma$ is transitive on $K \backslash\{p\}$.

For $\gamma \in \Gamma_{K}$ let $\Sigma^{\gamma}=\gamma \Sigma \gamma^{-1}$. Then $\Sigma$ and $\Sigma^{\gamma}$ are two connected 3-dimensional sugbroups of $\Gamma_{K}$ and $\Sigma T_{K}=\Sigma^{\gamma} T_{K}$. Since $T_{K}$ acts trivially on $K$, we have for the orbit $\Sigma(\gamma(p))=\Sigma T_{K}(\gamma(p))=\Sigma^{\gamma} T_{K}(\gamma(p))=\Sigma^{\gamma}(\gamma(p))=\gamma(p)$. This shows that $\gamma(p)$ is a fixed point of $\Sigma$. But $p$ is the only fixed point of $\Sigma$ on $K$. Therefore $\gamma(p)=p$. This proves that $p$ is a fixed point of $\Gamma_{K}$.

Finally, $\Gamma_{K}$ occurs as a subgroup of the collineation group of $\mathcal{B}^{\prime}$. Therefore, $\Gamma_{K}$ is solvable by Theorem 3.2.2. Now $\Gamma$, being the semidirect product of $\Gamma_{K}$ and $\Delta$, itself is solvable.

4.7. Remark. Suppose that the automorphism group $\Gamma$ of a 4-dimensional elation Laguerre plane $\mathcal{L}$ is 10-dimensional. Let $K$ be a circle of $\mathcal{L}$. Let $\Sigma$ be the connected component of the identity in the group $\left\{\gamma \in \Gamma_{K} \mid \operatorname{det}(\gamma)=1\right\}$. Then $\Sigma$ is a closed, connected 3-dimensional subgroup of $\Gamma_{K}^{1}$ and has discrete intersection with $T$. Furthermore, $\Gamma_{K}^{1}$ is the semidirect product of $\Sigma$ and $\left(\Gamma_{K} \cap T\right)^{1}$ and $\Sigma$ is locally isomorphic to $\Gamma^{1} /\left(\Gamma^{1} \cap T\right)$. Hence the above theorem describes the possible structure of a 10-dimensional group of automorphisms of an elation Laguerre plane.

\section{Elation Laguerre planes admitting simple Lie groups of automorphisms}

In this section we study 4-dimensional elation Laguerre planes that admit simple Lie groups of automorphisms. We shall show that precisely the semi-classical Laguerre planes admit such groups. The remaining situations occuring in Theorem 4.6, that is, non-simple 3-dimensional groups of automorphisms having a discrete intersection with the kernel, will be investigated in a later paper.

5.1. Lemma. Let $\mathcal{L}$ be a 4-dimensional elation Laguerre plane that admits a simple Lie group of automorphisms. Then $\mathcal{L}$ is classical or $\mathcal{L}$ admits a group $\Sigma$ isomorphic to $\mathrm{SO}_{3}(\mathbb{R})$ or $P S L_{2}(\mathbb{R})$ in the stabilizer of a circle. In the latter case, $\Sigma$ acts reducibly on the elation group by conjugation and $\Delta$ is the direct sum of two irreducible $\Sigma$-invariant 3-dimensional subspaces.

Proof. Since $T$ is solvable, a simple group $\Sigma$ of automorphisms of $\mathcal{L}$ must have trivial intersection with $T$. Hence the automorphism group $\Gamma$ of $\mathcal{L}$ has dimension at least $\operatorname{dim} \Sigma+\operatorname{dim} T \geq \operatorname{dim} \Sigma+7$. By Theorem 3.6, $\mathcal{L}$ is classical if $\operatorname{dim} \Sigma \geq 4$. However, every simple Lie groups has dimension at least 3 and a simple 3-dimensional Lie group is isomorphic to either $\mathrm{SO}_{3}(\mathbb{R})$ or $\mathrm{PSL}_{2}(\mathbb{R})$.

By Lemma 4.1 the group $\Sigma$ is isomorphic to a subgroup in the stabilzer of a circle. Hence we are in the situation of Theorem 4.6. As seen there, $\Delta$ is the direct sum of two irreducible $\Sigma$-invariant 3-dimensional subspaces.

5.2. Both groups $\mathrm{SO}_{3}(\mathbb{R})$ and $\mathrm{PSL}_{2}(\mathbb{R})$ occur as groups of automorphisms of the classical complex Laguerre plane $\tilde{\mathcal{L}}$. We describe $\tilde{\mathcal{L}}$ as in 2.11 . In fact, $\mathcal{L}_{0}$ is the classical complex Laguerre plane. The point set of $\tilde{\mathcal{L}}$ is $\tilde{P}=(\mathbb{C} \cup\{\infty\}) \times \mathbb{C}$. Two 
points $(z, w)$ and $\left(z^{\prime}, w^{\prime}\right)$ are parallel if and only if $z=z^{\prime}$. Circles of $\tilde{\mathcal{L}}$ are of the form

$$
K_{a_{2}, a_{1}, a_{0}}=\left\{(z, w) \in \mathbb{C} \times \mathbb{C} \mid w=a_{2} z^{2}+a_{1} z+a_{0}\right\} \cup\left\{\left(\infty, a_{2}\right)\right\}
$$

for $a_{0}, a_{1}, a_{2} \in \mathbb{C}$. The stabilizer of the circle $K_{0,0,0}$ contains the following automorphisms

$$
(z, w) \mapsto \begin{cases}\left(\frac{a z+b}{c z+d}, \frac{w}{(c z+d)^{2}}\right), & \text { for } z \in \mathbb{C}, c z+d \neq 0 \\ \left(\infty, c^{2} w\right), & \text { for } z \in \mathbb{C}, c z+d=0 \\ \left(\frac{a}{c}, \frac{w}{c^{2}}\right), & \text { for } z=\infty, c \neq 0 \\ \left(\infty, d^{2} w\right), & \text { for } z=\infty, c=0\end{cases}
$$

for all $a, b, c, d \in \mathbb{C}, a d-b c=1$. The collection of all these automorphisms forms a group isomorphic to the simple Lie group $\mathrm{PSL}_{2}(\mathbb{C})$. On the set of circles

$$
\tilde{\mathcal{C}}=\left\{K_{a_{2}, a_{1}, a_{0}} \mid a_{0}, a_{1}, a_{2} \in \mathbb{C}\right\}
$$

of $\tilde{\mathcal{L}}$ this group acts as follows. The coordinate vector $v \in \mathbb{C}^{3}$ of a circle $K_{v}$ is mapped to $v \cdot\left(\begin{array}{ccc}d^{2} & -2 b d & b^{2} \\ -c d & a d+b c & -a b \\ c^{2} & -2 a c & a^{2}\end{array}\right)$. In fact, this is, up to equivalence, the unique irreducible 3-dimensional faithful complex representation $\alpha$ of $\mathrm{PSL}_{2}(\mathbb{C})$.

Let $\beta: \mathrm{SL}_{2}(\mathbb{C}) \rightarrow \mathrm{PSL}_{2}(\mathbb{C})$ denote the canonical projection. (This is a twofold covering map of $\mathrm{PSL}_{2}(\mathbb{C})$.) Then the above formula describes how $\beta\left(\left(\begin{array}{ll}a & b \\ c & d\end{array}\right)\right)$ operates on $\mathbb{C}^{3}$. Let $\tilde{\Sigma}_{-1}$ and $\tilde{\Sigma}_{1}$ be the following subgroups of $\mathrm{PSL}_{2}(\mathbb{C})$

$$
\begin{aligned}
\tilde{\Sigma}_{-1} & =\left\{\left.\beta\left(\left(\begin{array}{cc}
a & \bar{b} \\
-b & \bar{a}
\end{array}\right)\right)|a, b \in \mathbb{C},| a\right|^{2}+|b|^{2}=1\right\} \text { and } \\
\tilde{\Sigma}_{1} & =\left\{\left.\beta\left(\left(\begin{array}{cc}
a & \bar{b} \\
b & \bar{a}
\end{array}\right)\right)|a, b \in \mathbb{C},| a\right|^{2}-|b|^{2}=1\right\} .
\end{aligned}
$$

Then $\tilde{\Sigma}_{-1}$ and $\tilde{\Sigma}_{1}$ are isomorphic to $\mathrm{SO}_{3}(\mathbb{R})$ and $\mathrm{PSL}_{2}(\mathbb{R})$, respectively. We restrict the representation $\alpha$ to $\tilde{\Sigma}_{-1}$ and $\tilde{\Sigma}_{1}$, i.e.,

$$
\alpha\left(\beta\left(\left(\begin{array}{cc}
a & \bar{b} \\
\epsilon b & \bar{a}
\end{array}\right)\right)\right)=\left(\begin{array}{ccc}
\bar{a}^{2} & -2 \bar{a} \bar{b} & \bar{b}^{2} \\
-\epsilon \bar{a} b & |a|^{2}+\epsilon|b|^{2} & -a \bar{b} \\
b^{2} & -2 \epsilon a b & a^{2}
\end{array}\right)
$$

where $\epsilon= \pm 1$. This yields faithful linear real representations of $\mathrm{SO}_{3}(\mathbb{R})$ and $\mathrm{PSL}_{2}(\mathbb{R})$ on $\mathbb{C}^{3} \cong \mathbb{R}^{6}$ and $\mathbb{C}^{3}$ is the direct sum of the invariant irreducible 3 -dimensional real subspaces $\{(z, i s,-\epsilon \bar{z}) \mid z \in \mathbb{C}, s \in \mathbb{R}\}$ and $\{(z, r, \epsilon \bar{z}) \mid z \in \mathbb{C}, r \in \mathbb{R}\}$.

Both groups act on $K_{0,0,0}$ like in Theorem 4.6. $\tilde{\Sigma}_{-1}$ is transitive on $K_{0,0,0}$ and $\tilde{\Sigma}_{1}$ has the 1-dimensional orbit $\tilde{B}_{1}=\{(z, 0)|z \in \mathbb{C}| z \mid,=1\}$ and the two 2-dimensional orbits $\{(z, 0)|z \in \mathbb{C}| z \mid,<1\}$ and $\{(z, 0)|z \in \mathbb{C}| z \mid,>1\} \cup\{(\infty, 0)\}$. Furthermore, the stabilizer in both groups of $(\infty, 0)$ is isomorphic to $\mathrm{SO}_{2}(\mathbb{R})$ and is represented under $\alpha$ as the group $\left\{\left(\begin{array}{lll}a & 0 & 0 \\ 0 & 1 & 0 \\ 0 & 0 & \bar{a}\end{array}\right)|a \in \mathbb{C}| a \mid,=1\right\}$. 
5.3. Let $\Sigma$ be a subgroup in the stabilzer of the circle $K$ and assume that $\Sigma$ is isomorphic to either $\mathrm{SO}_{3}(\mathbb{R})$ or $\mathrm{PSL}_{2}(\mathbb{R})$. Let $\Phi$ be the semidirect product of $\Sigma$ and $\Delta$. It follows from Theorem 4.6 that $\Phi$ is transitive on the points of $\mathcal{L}$ in case $\Sigma \cong \mathrm{SO}_{3}(\mathbb{R})$ and that $\Phi$ has three orbits on the point set of $\mathcal{L}$ in case $\Sigma \cong \mathrm{PSL}_{2}(\mathbb{R})$, one 3-dimensional orbit and two open 4-dimensional orbits. Let $Z$ be a 4-dimensional orbit of $\Phi$. (Note that $Z=P$ in case $\Sigma \cong \mathrm{SO}_{3}(\mathbb{R})$.) Furthermore, let $\mathcal{Z}$ the geometry induced by $\mathcal{L}$ on $Z$, that is, $\mathcal{Z}$ has point set $Z$ and the circles of $\mathcal{Z}$ are of the form $Z \cap C$ for all circles $C \in \mathcal{C}$. Then $\Phi$ is flag transitive on $\mathcal{Z}$.

According to $[14, \S 2]$ the geomtry of $\mathcal{Z}$ can be reconstructed from the group $\Phi$ in the following way. We choose a point $p \in K \cap Z$. Then the points of $\mathcal{Z}$ correspond to the cosets $\gamma \Phi_{p}$ of the stabilizer $\Phi_{p}$ of $p$ in $\Phi$ and the circles of $\mathcal{Z}$ correspond to the cosets $\delta \Phi_{K}$ of the stabilizer $\Phi_{K}$ of $K$ in $\Phi$. A point $q \sim \gamma \Phi_{p}$ is incident with the circle $L \sim \delta \Phi_{K}$ if and only if the two coset have non-empty intersection, i.e., $\gamma \Phi_{p} \cap \delta \Phi_{K} \neq \emptyset$.

We consider the corresponding subgroups $\tilde{\Sigma}, \tilde{\Delta}$ and $\tilde{\Phi}$ in the automorphism group $\tilde{\Gamma}$ of the classical complex Laguerre plane $\tilde{\mathcal{L}}$, that is, $\tilde{\Sigma}$ equals $\tilde{\Sigma}_{-1}$ or $\tilde{\Sigma}_{1}$ from 5.2 and $\tilde{\Delta}$ is the collection of all automorphisms

$$
(z, w) \mapsto \begin{cases}\left(z, w+b_{2} z^{2}+b_{1} z+b_{0}\right), & \text { for } z \in \mathbb{C} \\ \left(\infty, w+b_{2}\right), & \text { for } z=\infty\end{cases}
$$

We form the corresponding subgeometry $\tilde{\mathcal{Z}}$, that is, $\tilde{\mathcal{Z}}=\tilde{\mathcal{L}}$ or $\tilde{\mathcal{Z}}$ is the subgeometry induced on $\tilde{Z}=\left\{(z, w) \in \mathbb{C}^{2}|| z \mid>1\right\} \cup\{\infty\} \times \mathbb{C}$.

The action of $\Sigma$ on $\Delta$ by conjugation is equivalent to the corresponding action of $\tilde{\Sigma}$ on $\tilde{\Delta}$. This implies that $\Phi=\Sigma \Delta$ and $\tilde{\Phi}=\tilde{\Sigma} \tilde{\Delta}$ are isomorphic as topological groups. Furthermore, a continuous isomorphism $\gamma: \Phi \rightarrow \tilde{\Phi}$ can be chosen such that $\gamma(\Sigma)=\tilde{\Sigma}$ and $\gamma(\Delta)=\tilde{\Delta}$

Our aim is to identify $\Phi_{p}$ with $\tilde{\Phi}_{\tilde{p}}$ for a suitable point $\tilde{p} \in \tilde{Z}$ under the isomorphism $\gamma$. We can then identify $\mathcal{Z}$ with the corresponding subgeometry $\tilde{\mathcal{Z}}$ of the classical complex Laguerre plane.

5.4. Let $p$ and $q$ be points on $K$. We denote the bundle of all circles through $p$ by

$$
\mathcal{C}_{p}=\{L \in \mathcal{C} \mid p \in L\}
$$

Furthermore, let

$$
\mathcal{C}_{p, q}=\mathcal{C}_{p} \cap \mathcal{C}_{q}
$$

denote the pencil of circles through $p$ and $q$ for $p \neq q$ and

$$
\mathcal{C}_{p, p}=\{L \in \mathcal{C} \mid L \text { touches } K \text { at } p\}
$$

denotes the tangent pencil to $K$ at $p$.

Under the usual identification of $\Delta$ with $\mathcal{C}$, the spaces $\mathcal{C}_{p}$ and $\mathcal{C}_{p, q}$ for $p \neq q$ correspond to $\Delta_{p}$ and $\Delta_{p, q}$, respectively. These spaces are therefore vector subspaces of $\mathcal{C} \cong \mathbb{R}^{6}$ of respective dimensions 4 and 2 . Using the matrix representation of 
4-dimensional elation Laguerre planes from 2.10 , one also sees that $\mathcal{C}_{p, p}$ is a 2 -dimensional subspace of $\mathcal{C}$.

Let $p \in K \cap Z$. The stabilizer $\Sigma_{p}$ is isomorphic to $\mathrm{SO}_{2}(\mathbb{R})$ and there is precisely one $p^{\prime} \in K, p^{\prime} \neq p$, that is also fixed by $\Sigma_{p}$. Obviously, $\Sigma_{p}$ leaves the spaces $\mathcal{C}_{p}$, $\mathcal{C}_{p, p^{\prime}}$ and $\mathcal{C}_{p, p}$ invariant and $\mathcal{C}_{p}$ is the direct sum of $\mathcal{C}_{p, p^{\prime}}$ and $\mathcal{C}_{p, p}$. We want to characterize $\mathcal{C}_{p}$ by the action of $\Sigma_{p}$ on $\Delta \simeq \mathcal{C}$ as this determines $\Delta_{p}$.

Lemma. Let $p \in K \cap Z$ and let $p^{\prime} \in K$ be the second fixed point of $\Sigma_{p} \cong S O_{2}(\mathbb{R})$. Then $\Sigma_{p}$ acts trivially on $\mathcal{C}_{p, p^{\prime}}$.

Proof. Let $U$ be a 3-dimensional irreducible $\Sigma$-invariant subspace of $\mathcal{C}$. Let $U_{q}=$ $U \cap \mathcal{C}_{q}$ and $U_{q, r}=U \cap \mathcal{C}_{q, r}$ for $q, r \in K$. Note that $\sigma\left(U_{q}\right)=U_{\sigma(q)}$ and $\sigma\left(U_{q, r}\right)=$ $U_{\sigma(q), \sigma(r)}$ for all $\sigma \in \Sigma$.

We first claim that $\operatorname{dim} U_{q, r} \leq 1$ for all $q, r \in K$. Since $\Sigma$ fixes no point on $K$, there is a $\sigma \in \Sigma$ such that $\sigma(q) \neq q, r$. It then follows from the uniqueness of joining or touching that $\mathcal{C}_{q, r} \cap \mathcal{C}_{\sigma(q), \sigma(r)}=\{K\}$. Using the dimension formula for vector spaces, we therefore find

$$
\begin{aligned}
2 \operatorname{dim} U_{q, r} & =\operatorname{dim} U_{q, r}+\operatorname{dim} U_{\sigma(q), \sigma(r)} \\
& =\operatorname{dim}\left(U_{q, r}+U_{\sigma(q), \sigma(r)}\right)+\operatorname{dim}\left(U_{q, r} \cap U_{\sigma(q), \sigma(r)}\right) \\
& \leq \operatorname{dim} U+0 \\
& =3
\end{aligned}
$$

This proves our claim.

We now show that $1 \leq \operatorname{dim} U_{q} \leq 2$. With $\sigma \in \Sigma$ as before we analoguously obtain

$$
\begin{aligned}
2 \operatorname{dim} U_{q} & =\operatorname{dim} U_{q}+\operatorname{dim} U_{\sigma(q)} \\
& =\operatorname{dim}\left(U_{q}+U_{\sigma(q)}\right)+\operatorname{dim}\left(U_{q} \cap U_{\sigma(q)}\right) \\
& \leq \operatorname{dim} U+\operatorname{dim} U_{q, \sigma(q)} \\
& \leq 4
\end{aligned}
$$

The left-hand inequality follows from

$$
\operatorname{dim} U_{q}=\operatorname{dim}\left(U \cap \mathcal{C}_{q}\right)=\operatorname{dim} U+\operatorname{dim} \mathcal{C}_{q}-\operatorname{dim}\left(U+\mathcal{C}_{q}\right) \geq 3+4-6=1
$$

We finally show that $\operatorname{dim} U_{q}=1$ for every point $q$ having a 2-dimensional orbit under $\Sigma$, i.e., $q \in K \backslash B_{1}$ where $B_{1}$ denotes the empty set in case $\sigma \cong \mathrm{SO}_{3}(\mathbb{R})$ or the 1 -dimensional orbit in case $\sigma \cong \mathrm{PSL}_{2}(\mathbb{R})$. Since $q \notin B_{1}$, the stabilizer $\Sigma_{q}$ is isomorphic to $\mathrm{SO}_{2}(\mathbb{R})$ and there is precisely one $q^{\prime} \in K, q^{\prime} \neq q$, that is also fixed by $\Sigma_{q}$. Suppose that there is a $q \in K \backslash B_{1}$ such that $\operatorname{dim} U_{q}=2$. The stabilizer $\Sigma_{q} \cong \mathrm{SO}_{2}(\mathbb{R})$ of $q$ acts effectively as a group of rotations on $U \cong \mathbb{R}^{3}$ and by [18, 6.7.1] the space $V$ of fixed points is 1-dimensional. $V$ cannot be contained in $U_{q}$ for otherwise $\Sigma_{q}$ operates trivial on $U_{q}$ and $U_{q}$ is 2-dimensional. Since $L \cap K$ is invariant under $\Sigma_{q}$ for $L \in V$, it follows that $L \cap K \subseteq\left\{q, q^{\prime}\right\}$ for $L \in V, L \neq K$. This implies $V \subset U_{q, q} \cup U_{q, q^{\prime}} \cup U_{q^{\prime}, q^{\prime}}$ and therefore $V=U_{q^{\prime}, q^{\prime}}$. 
We now choose $\sigma \in \Sigma$ such that $\sigma\left(q^{\prime}\right) \neq q^{\prime}$ and let $r=\sigma\left(q^{\prime}\right)$. Then $\operatorname{dim} U_{r, r}=$ $\operatorname{dim} U_{q^{\prime}, q^{\prime}}=1$. Let $L \in U_{r, r}$ and $L^{\prime} \in U_{q^{\prime}, q^{\prime}}, L, L^{\prime} \neq K$. In the matrix representation 2.10 of $\mathcal{L}$ the circles $K, L$ and $L^{\prime}$ have coordinate vectors $0, c$ and $c^{\prime}$, respectively. We now form the circles $L_{s}, s \in \mathbb{R}$, whose coordinate vectors are $c-s c^{\prime}$. Then $L_{s} \in U, L_{0}=L$ and $L_{s}$ converges to $L$ as $s$ tends to 0 . By the continuity of intersection in $\mathcal{L}$ we obtain that $L_{s} \cap K$ converges to $L \cap K=\{r\}$ for $s \rightarrow 0$. In particular, $L_{s} \cap K$ is entirely contained in the orbit of $r$ under $\Sigma$. We can also find $L_{s} \cap K$ in the following way. Let $M_{s}$ be the circle with coordinate vector $s c^{\prime}$. Then $M_{s}, s \neq 0$, touches $K$ at $L^{\prime} \cap K=\left\{q^{\prime}\right\}$. Moreover, given the vector space structure of $\mathcal{C}$, we have $L_{s} \cap K=L \cap M_{s}$. In the derived projective plane $\mathcal{P}_{q^{\prime}}$ of $\mathcal{L}$ at $q^{\prime}$ the oval induced by $L$ has exactly two tangents through the infinite point $\omega_{K}$ of the line $K^{\prime}$ induced by $K$; namely $K^{\prime}$ and the line at infinity $W$. Since the line induced by $M_{s}$ also passes through $\omega_{K}$, we see that $L_{s} \cap K$ consists of two points for sufficiently small $s \neq 0$.

Let $L_{s} \cap K=\{x, y\}$ for $s$ sufficiently close to 0 . As seen above, there is a $\tau \in \Sigma$ such that $\tau(x)=r$. Hence $U_{r} \neq U_{r, r^{\prime}}$ where $r^{\prime} \in K$ is the second fixed point of $\Sigma_{r}$. Therefore $\operatorname{dim} U_{q^{\prime}}=\operatorname{dim} U_{r}=2$. (Note that in case $\Sigma \cong \mathrm{SO}_{3}(\mathbb{R})$ we could have obtained that $U_{q^{\prime}}$ is 2-dimensional directly with the transitivity of $\Sigma$ on $K$; however, in case $\Sigma \cong \mathrm{PSL}_{2}(\mathbb{R})$ the points $q$ and $q^{\prime}$ are in different orbits of $\Sigma$.) We now have

$$
\begin{aligned}
1 & \geq \operatorname{dim} U_{q, q^{\prime}} \\
& =\operatorname{dim}\left(U_{q} \cap U_{q^{\prime}}\right) \\
& =\operatorname{dim} U_{q}+\operatorname{dim} U_{q^{\prime}}-\operatorname{dim}\left(U_{q}+U_{q^{\prime}}\right) \\
& \geq 2+2-3=1 .
\end{aligned}
$$

Therefore $\operatorname{dim} U_{q, q^{\prime}}=1$. Furthermore, $\Sigma_{q}=\Sigma_{q, q^{\prime}}$ leaves $U_{q, q^{\prime}}$ invariant and must act trivially on this 1-dimensional subpace. This contradicts our previous observation that the 1-dimensional space $V$ of fixed points equals $U_{q^{\prime}, q^{\prime}}$. This shows that $\operatorname{dim} U_{q}=1$ for every point $q \in K \backslash B_{1}$.

Now let $p \in K \cap Z$. Then $p, p^{\prime} \in K \backslash B_{1}$ and $\operatorname{dim} U_{p}=\operatorname{dim} U_{p^{\prime}}=1$. Suppose that $U_{p} \neq U_{p^{\prime}}$. Then $\Sigma_{p}$ operates trivially on the 1-dimensional subspaces $U_{p}$ and $U_{p^{\prime}}$, thus on the 2-dimensional space $U_{p} \oplus U_{p^{\prime}}$ and consequently on the 3 -dimensional space $U$. Hence $U_{p}=U_{p^{\prime}}=U_{p, p^{\prime}}$ and $\Sigma_{p}$ operates trivially on $U_{p, p^{\prime}}$. Since $U_{p, p^{\prime}} \leq \mathcal{C}_{p, p^{\prime}} \cong \mathbb{R}^{2}$, the group $\Sigma_{p}$ must also operate trivially on $\mathcal{C}_{p, p^{\prime}}$.

5.5. Lemma. Let $\Psi \cong S \mathrm{O}_{2}(\mathbb{R})$ be the following group of matrices

$$
\Psi=\left\{\left(\begin{array}{lll}
\lambda & 0 & 0 \\
0 & 1 & 0 \\
0 & 0 & \bar{\lambda}
\end{array}\right)|\lambda \in \mathbb{C},| \lambda \mid=1\right\}
$$

acting naturally on $\mathbb{C}^{3}$. The only $\Psi$-invariant 2-dimensional real subspaces of $\mathbb{C}^{3} \cong$ $\mathbb{R}^{6}$ are

$$
\begin{aligned}
& -V=\{(0, z, 0) \mid z \in \mathbb{C}\} \\
& -V_{\infty}=\{(0,0, z) \mid z \in \mathbb{C}\} \text { and } \\
& -V_{c}=\{(z, 0, c \bar{z}) \mid z \in \mathbb{C}\} \text { for } c \in \mathbb{C}
\end{aligned}
$$


Furthermore, $V$ is the only 2-dimensional real subspace on which $\Psi$ acts trivially.

Proof. It readily follows that the above subspaces are 2-dimensional and $\Psi$-invariant and that among these spaces only $V$ is fixed pointwise. Conversely, let $U$ be a 2-dimensional $\Psi$-invariant real subspace of $\mathbb{C}^{3}$ and let $(a, b, c)$ be a non-zero vector in $U$. Since $U$ is $\Psi$-invariant, we have that, in particular, $(-a, b,-c) \in U$ and thus $(a, 0, c) \in U$ and $(0, b, 0) \in U$. Again by the invariance of $U$ and because $U$ is closed under scalar multiplication by reals, we see that $\{(z a, 0, \bar{z} c) \mid z \in \mathbb{C}\} \subseteq U$. However, this set is a real vector subspace of dimension 2 unless $a=c=0$. Furthermore, if $a=0$ and $c \neq 0$, we obtain the space $V_{\infty}$; for $a \neq 0$ we obtain the space $V_{c \bar{a}-1}$. In each case $b=0$ and $U$ equals one of the spaces $V_{d}$ for some $d \in \mathbb{C} \cup\{\infty\}$. The same argument further shows that if $U$ is not of this form the first and third entries in each vector in $U$ must be equal to 0 . Hence $U=V$ in this case.

5.6. Lemma. Let $p \in K \cap Z$. Then, up to equivalence under the action of $\Sigma, \mathcal{C}_{p}$ corresponds to the subspace $V+V_{\infty}$.

Proof. By Lemmata 5.4 and 5.5 the pencil $\mathcal{C}_{p, p^{\prime}}$, where $p^{\prime}$ is the second fixed point of $\Sigma_{p}$ on $K$, must correspond to the subspace $V$ of $\mathbb{C}^{3}$. Hence $\mathcal{C}_{p, p}$ must correspond to one of the subspaces $V_{c}$ for $c \in \mathbb{C} \cup\{\infty\}$. Since $\mathcal{C}_{p}=\mathcal{C}_{p, p^{\prime}} \oplus \mathcal{C}_{p, p}$, we obtain that $\mathcal{C}_{p}$ must then correspond to $V+V_{c}$. So we have to show that $c=\infty$ up to equivalence.

We assume that $c \neq \infty$, i.e., $c \in \mathbb{C}$. Recall that, up to equivalence, the action of $\Sigma$ on $\mathcal{C}$ is given by the representation $\alpha$ of $\Sigma$ on $\mathbb{C}^{3}$, see 5.2 . It is easily verified that $\alpha(\sigma)\left(V_{c}\right) \subseteq V+V_{c}$ for each $\sigma \in \Sigma$ if $|c|=1$. However, this implies $\Sigma\left(\mathcal{C}_{p, p}\right) \subseteq \mathcal{C}_{p}$, and $p$ is a fixed point of $\Sigma$ in contradiction to Theorem 4.6. Therefore $|c| \neq 1$. Then $\theta_{c}: \mathbb{C}^{3} \rightarrow \mathbb{C}^{3}$ defined by

$$
\theta_{c}\left(z_{1}, z_{2}, z_{3}\right)=\left(c \bar{z}_{1}-z_{3}, c \bar{z}_{2}-\epsilon z_{2}, c \bar{z}_{3}-z_{1}\right)
$$

is an automorphism of the real vector space $\mathbb{C}^{3}$. (The inverse of $\theta_{c}$ is $\frac{1}{1-|c|^{2}} \theta_{-c}$.) Furthermore, $\theta_{c}$ maps $V_{c}$ to $V_{\infty}$ and $V$ into itself. Finally, conjugation by $\theta_{c}$ takes $\alpha(\sigma)$ for $\sigma \in \Sigma$ to $\alpha(\sigma)^{-1}$ so that $\alpha(\Sigma)$ is invariant under conjugation by $\theta_{c}$. Hence, changing the coordinates of $\mathbb{C}^{3}$, we can assume $c=\infty$, i.e., that $\mathcal{C}_{p}$ corresponds to $V+V_{\infty}$

5.7. We are now in a position to identify $\Phi_{p}$ in $\Phi$, see 5.3 .

Proposition. Let $p \in K \cap Z$. Then there is an isomorphism from $\Phi$ to $\tilde{\Phi}$ that takes $\Sigma$ to $\tilde{\Sigma}$ and $\Phi_{p}$ to $\tilde{\Phi}_{(\infty, 0)}$. Hence $\mathcal{Z}$ and $\tilde{\mathcal{Z}}$ are isomorphic geometries. In particular, $\mathcal{L}$ is classical in case $\Sigma \cong \mathrm{SO}_{3}(\mathbb{R})$.

Proof. As seen in 5.3 there is an isomorphism $\gamma: \Phi \rightarrow \tilde{\Phi}$ such that $\gamma(\Sigma)=\tilde{\Sigma}$ and $\gamma(\Delta)=\tilde{\Delta}$. Now $\gamma\left(\Delta_{p}\right)$ is a 4-dimensional subspace of $\tilde{\Delta}$ and it is invariant under $\gamma\left(\Delta_{p}\right) \cong \mathrm{SO}_{2}(\mathbb{R})$. We may assume that $\gamma\left(\Delta_{p}\right)$ acts on $\tilde{\Delta}$ like $\Psi$ on $\mathbb{C}^{3}$. By the preceding lemma we can find a coordinate system of $\mathbb{C}^{3}$ such that $\mathcal{C}_{p}$ and thus $\Delta_{p}$ corresponds to $V+V_{\infty}$. In the classical complex Laguerre plane $\tilde{\mathcal{L}}$ this space describes the bundle of circles through the point $(\infty, 0)$. More precisely, as seen 
in the proof of Lemma 5.6, the operaton $\kappa$ of conjugation by $\theta_{c}$, where $\theta_{\infty}=i d$, yields $\kappa \gamma\left(\Delta_{p}\right)=\tilde{\Delta}_{(\infty, 0)}$. Since $\kappa$ is compatible with the restriction of $\gamma$ to $\Sigma$, the composition $\gamma^{\prime}=\kappa \gamma$ is an isomorphism as claimed. Since the geometry of $\mathcal{Z}$ is uniquely determined by $\Phi$, cf. 5.3 , and likewise for $\tilde{\mathcal{Z}}$, we obtain that $\mathcal{Z}$ and $\tilde{\mathcal{Z}}$ are isomorphic geometries; an isomorphism is induced by $\gamma^{\prime}$ taking $p$ to $(\infty, 0)$ and $K$ to $K_{0,0,0}$.

If $\Sigma \cong \mathrm{SO}_{3}(\mathbb{R})$, then we have $\mathcal{Z}=\mathcal{L}$ and $\tilde{\mathcal{Z}}=\tilde{\mathcal{L}}$, so $\mathcal{L}$ is classical.

Corollary. A 4-dimensional elation Laguerre plane $\mathcal{L}$ admitting a simple Lie group of automorphisms is semi-classical.

Proof. By Lemma 5.1 the Laguerre plane $\mathcal{L}$ is classical and thus semi-classical or we are in the situation described in 5.3. By the preceding proposition $\mathcal{Z}$ and $\tilde{\mathcal{Z}}$ are isomorphic geometries, that is, $\mathcal{Z}$ can be identified with the geometry induced by the classical complex Laguerre plane on a corresponding set of points. In case $\Sigma \cong \mathrm{SO}_{3}(\mathbb{R})$, we already know that $\mathcal{L}$ is classical by Proposition 5.7 . In case $\Sigma \cong \mathrm{PSL}_{2}(\mathbb{R})$, there is exactly one other 4-dimensional orbit $Z^{\prime}$ of $\Phi$. We form the corresponding induced geometry $\mathcal{Z}^{\prime}$. Then $\mathcal{Z}$ and $\mathcal{Z}^{\prime}$ are isomorphic to geometries induced by the classical complex Laguerre plane on corresponding sets of points. We form the topological closure $\bar{Z}$ and $\overline{Z^{\prime}}$ of $Z$ and $Z^{\prime}$, respectively, in the point set $P$ of $\mathcal{L}$. Obviously, $\bar{Z}$ is the union of $Z$ and the 3 -dimensional orbit $B_{3}$ of $\Phi$, i.e., all points that are parallel to a point in $B_{1}$, and likewise for $\overline{Z^{\prime}}$. The induced geometries $\overline{\mathcal{Z}}$ and $\overline{\mathcal{Z}^{\prime}}$ are still isomorphic to subgeometries of $\tilde{\mathcal{L}}$ since the geometries of $\overline{\mathcal{Z}}$ and $\overline{\mathcal{Z}^{\prime}}$ are uniquely determined by the geometries of $\mathcal{Z}$ and $\mathcal{Z}^{\prime}$. Now, $\bar{Z} \cup \overline{Z^{\prime}}=P, \bar{Z}$ and $\overline{Z^{\prime}}$ are closed subsets of $P$, they are closed with respect to the parallelism of $\mathcal{L}$ and $\bar{Z} \cap \overline{Z^{\prime}} \cap K$ is a closed Jordan curve in the circle $K$. Hence $\mathcal{L}$ is a semi-classical Laguerre plane by the definition given in $[29,2.4]$ and it is isomorphic to one of the Laguerre planes described in 2.11, cf. [29, Theorem B].

5.8. In the theorem below we give various characterizations of the semi-classical Laguerre planes in terms of their automorphism groups.

Theorem. Let $\mathcal{L}$ be a 4-dimensional elation Laguerre plane with automorphism group $\Gamma$. Then the following statements are equivalent.

(1) There is a circle $K$ and a closed, connected 3-dimensional group $\Sigma \leq \Gamma_{K}$ that has discrete intersection with the kernel $T$ such that $\Sigma$ acts fixed-pointfree on $K$ and reducibly on $\mathcal{C}$;

(2) $\Gamma$ fixes no parallel class and the stabilizer of a circle contains a 4-dimensional subgroup that fixes a non-trivial proper subspace of $\mathcal{C}$;

(3) $\Gamma$ is non-solvable and the stabilizer of a circle contains a 4-dimensional subgroup that fixes a non-trivial proper subspace of $\mathcal{C}$;

(4) $\mathcal{L}$ admits a simple Lie group of automorphisms;

(5) $\mathcal{L}$ is semi-classical.

Proof. As seen in 2.11, a semi-classical Laguerre plane admits a group of automorphisms isomorphic to $\mathbb{R} \times \mathrm{PSL}_{2}(\mathbb{R})$ in the stabilizer of the circle $K_{0,0,0}$ and the action of $\mathrm{PSL}_{2}(\mathbb{R})$ on $K_{0,0,0}$ and thus on $\Pi$ is equivalent to the standard action of 
$\mathrm{PSL}_{2}(\mathbb{R})$ as a subgroup of $\mathrm{PSL}_{2}(\mathbb{R})$ on $\mathbb{S}_{2} \simeq \mathbb{C} \cup\{\infty\}$. Also, the action of $\mathrm{PSL}_{2}(\mathbb{R})$ on the set of circles is like in 5.2. This shows that a semi-classical Laguerre plane has all the properties stated in the theorem.

Conversely, (4) implies (5); this is just Corollary 5.7. Remark 4.7 and Theorem 4.6 show that (1) implies (2) and also that (2) implies (4). If $\Gamma$ is non-solvable, then it must contain a semisimple Lie group of automorphisms. Such a group has dimension at least 3 and we may assume that it fixes a circle. Thus (3) implies (2) by Theorem 4.6. Hence $\mathcal{L}$ is semi-classical in all these cases.

5.9. We finally give various characterizations of the classical complex Laguerre plane in terms of its automorphism group.

Theorem. Let $\mathcal{L}$ be a 4-dimensional elation Laguerre plane with automorphism group $\Gamma$ and kernel $T$. Then the following statements are equivalent.

(1) $\Gamma$ is flag-transitive;

(2) $\Gamma$ is transitive on the points of $\mathcal{L}$;

(3) $\Gamma$ is transitive on the set of parallel classes of $\mathcal{L}$;

(4) $\Gamma$ contains a compact group of dimension at least 2;

(5) $\Gamma$ is at least 11-dimensional;

(6) $T$ is 8-dimensional;

(7) $\mathcal{L}$ is classical.

Proof. The classical complex Laguerre plane $\tilde{\mathcal{L}}$ admits a group of automorphisms isomorphic to $\mathrm{SO}_{3}(\mathbb{R})$ in the stabilizer of the circle $K_{0,0,0}$. This group acts transitively on the set of parallel classes and on $K_{0,0,0}$. Together with the elation group we see that (1) - (4) are satisfied. Furthermore, the automorphism group of $\tilde{\mathcal{L}}$ is 14-dimensional.

Conversely, Theorem 3.6 shows that (5) implies (7), and (6) implies (7) by [30, Proposition 2.8] or [8, 5.2]. Clearly, (1) implies (2) and (2) implies (3). If (3) is satisfied, then $\Gamma / T$ is transitive and effective on $\Pi \simeq \mathbb{S}_{2}$. Therefore $\operatorname{dim} \Gamma / T \geq 3$ by Theorem 2.6 .2 and $\operatorname{dim} \Gamma=\operatorname{dim} \Gamma / T+\operatorname{dim} T \geq 3+7=10$. If $\operatorname{dim} \Gamma \geq 11$, then $\mathcal{L}$ is classical by Theorem 3.6. If $\operatorname{dim} \Gamma=10$, then we are in the situation of Theorem 4.6 with $\Sigma \cong \mathrm{SO}_{3}(\mathbb{R})$. Hence $\mathcal{L}$ is classical by Proposition 5.7.

We now assume that $\Gamma$ contains a compact group $\Sigma$ of dimension at least 2 . We may assume that $\Sigma$ is connected. Since $\Delta \cong \mathbb{R}^{6}$, we see that $\Sigma$ is in the elation complement. By Lemma 4.1 we may therefore further assume that $\Sigma$ fixes a circle $K$. By [18, Theorem 6.7.1] a compact connected group acting effectively on $\mathbb{S}_{2}$ is isomorphic to and acts equivalently to either $\mathrm{SO}_{2}(\mathbb{R})$ or $\mathrm{SO}_{3}(\mathbb{R})$ in their standard actions on $\mathbb{S}_{2}$. This shows that either $\Sigma$ contains a subgroup isomorphic to $\mathrm{SO}_{3}(\mathbb{R})$ or $\Sigma /(T \cap \Sigma) \cong \mathrm{SO}_{2}(\mathbb{R})$. In the former case $\mathcal{L}$ is classical as seen before. In the latter case $T \cap \Sigma$ must be at least 1-dimensional. Furthermore, $T \cap \Sigma$ is compact, so that $T \cap \Sigma$ cannot be the 1-dimensional group of homotheties by real non-zero scalars that every elation Laguerre plane admits. Therefore $\operatorname{dim}(T \cap \Sigma) \geq 2$ and thus $\operatorname{dim} T=8$. Hence (6) is satisfied and $\mathcal{L}$ is classical. 


\section{REFERENCES}

1. R. Artzy and H. Groh, Laguerre and Minkowski planes produced by dilatations, J. Geom. 26 (1986), 1-20.

2. D. Betten, 4-dimensionale Translationsebenen, Math. Z. 128 (1972), 129-151.

3. D. Betten, 4-dimensionale Translationsebenen mit 8-dimensionaler Kollineationsgruppe, Geom. Dedicata 2 (1973), 327-339.

4. L.E.J. Brouwer, Die Theorie der endlichen kontinuierlichen Gruppen unabhängig von den Axiomen von Lie, Math. Ann. 67 (1909), 244-267.

5. T. Buchanan, Ovale und Kegelschnitte in der komplexen projektiven Ebene, Math. Phys. Sem. Ber. 26 (1979), 244-260.

6. T. Buchanan, H. Hähl and R. Löwen, Topologische Ovale, Geom. Dedicata 9 (1980), 401-424.

7. L.R.A. Casse, J.A. Thas and P.R. Wild, $\left(q^{n}+1\right)$-sets of $P G(3 n-1, q)$, generalized quadrangles and Laguerre planes, Simon Stevin 59 (1985), 21-42.

8. A. Förtsch, Kollineationsgruppen endlichdimensionaler topologischer Benzebenen, Dissertation, Erlangen, 1982.

9. H. Freudenthal and H. de Vries, Linear Lie groups, Academic Press, New York, 1969.

10. H. Groh, Topologische Laguerreebenen I, Abh. Math. Sem. Univ. Hamburg 32 (1968), $216-231$.

11. H. Groh, Topologische Laguerreebenen II, Abh. Math. Sem. Univ, Hamburg 34 (1970), 11-21.

12. H. R. Halder, Dimension der Bahnen lokalkompakter Gruppen, Arch. Math. 22 (1971), 302303.

13. H. R. Halder, Über Bahnen lokalkompakter Gruppen auf Flächen, Geom. Dedicata 2 (1973), 101-109.

14. D.G. Higman and J.E. McLaughlin, Geometric ABA-groups, Illinois J. Math. 5 (1961), 382397.

15. R. Löwen, Projectivities and the geometric structure of topological planes, in: Geometry - von Staudt's point of view (eds P. Plaumann and K. Strambach), Bad Windsheim (1981), 339-372.

16. R. Löwen, Topological pseudo-ovals, elation Laguerre planes, and elation generalized quadrangles, Math. Z. 216 (1994), 347-369.

17. R. Löwen and U. Pfüller, Two-dimensional Laguerre planes over convex functions, Geom. Dedicata 23 (1987), 73-85.

18. D. Montgomery and L. Zippin, Topological Transformation Groups, Wiley Interscience, New York, 1955.

19. G.D. Mostow, The extensibility of local Lie groups of transformations and groups on sufaces, Ann. Math. 52 (1950), 606-636.

20. S. E. Payne and J.A. Thas, Finite generalized quadrangles, Research Notes in Mathematics, vol. 110, Pitman, Boston, 1984.

21. H. Salzmann, Topological planes, Adv. Math. 2 (1967), 1-60.

22. H. Salzmann, Kollineationsgruppen kompakter vier-dimensionaler Ebenen, Math. Z. 117 (1970), 112-124.

23. H. Salzmann, Kollineationsgruppen kompakter 4-dimensionaler Ebenen II, Math. Z. 121 (1971), 104-110.

24. H. Salzmann, Kompakte vier-dimensionale projektive Ebenen mit 8-dimensionaler Kollineationsgruppe, Math. Z. 130 (1973), 235-247.

25. H. Salzmann, D. Betten, T. Grundhöfer, H. Hähl, R. Löwen and M. Stroppel, Compact projective planes, de Gruyter, Berlin, 1995.

26. A.E. Schroth, Topological circle planes and topological quadrangles, Pitman Research Notes in Mathematics Series, vol. 337, Longman, Essex, UK, 1995.

27. G.F. Steinke, The automorphism group of Laguerre planes, Geom. Dedicata 21 (1986), 55-58.

28. G.F. Steinke, Eine Klassifikation 4-dimensionaler Laguerre-Ebenen mit großer Automorphismengruppe, Habilitationsschrift, Kiel, 1987.

29. G.F. Steinke, Semiclassical 4-dimensional Laguerre planes, Forum Math. 2 (1990), $233-247$.

30. G.F. Steinke, The elation group of a 4-dimensional Laguerre plane, Monatsh. Math. 111 (1991), 207-231. 
31. G.F. Steinke, The point space of 4-dimensional Laguerre planes, Arch. Math. 56 (1991), 100-104.

32. G.F. Steinke, 4-dimensional point-transitive groups of automorphisms of 2-dimensional Laguerre planes, Result. Math. 24 (1993), 326-341.

33. G.F. Steinke, Topological circle geometries, Handbook of incidence geometry (Ed. F. Buekenhout), pp. 1325-1354, Elsevier, Amsterdam, 1995.

34. J.A. Thas, The $m$-dimensional projective space $S_{m}\left(M_{n}(G F(q))\right)$ over the total matrix algebra $M_{n}(G F(q))$ of $n \times n$ matrices with elements in the Galois field $G F(q)$, Rend. Mat. (6) 4 (1971), 459-532.

35. J. Tits, Sur certaines classes d'espaces homogènes de groupes de Lie, Acadèmie royale de Belgique - Mèmoires Tome XXIV, Fasc. 3, Bruxelles, 1955.

36. J. Tits, Sur les groupes doublement transitifs continus: corrections et compléments, Commentarii Math. Helvet. 33 (1956), 234-240.

37. J. Tits, Tabellen zu den einfachen Liegruppen und ihren Darstellungen, Lecture Notes in Mathematics, vol. 40, Springer, Heidelberg, 1967. 